\title{
Framework towards the Process of Estimating or Predicting Perceived QoE Based on the Datasets Obtained From the Mobile Network
}

\author{
Damera Priyanka, Mamidala Soujanya, Syed Abdul Moeed
}

\begin{abstract}
Nowadays, the research study community visualizes a standard shift that is going to put the focus on Quality of Experience metrics, which relate directly to complete consumer satisfaction. Yet, determining QoE coming from QoS sizes is a daunting job that powerful Software Defined Network operators are currently able to tackle through artificial intelligence strategies. In this paper, our experts pay attention to a few essential QoE factors, and we to begin with proposing a Bayesian Network design to anticipate re-buffering proportion. This paper suggested a structure for modeling mobile network QoE, making use of the vast records analytics approach. The planned platform explains the method of estimating or forecasting perceived QoE based upon the datasets obtained or collected from the mobile network to enable the mobile network operators efficiently to deal with the network functionality as well as supply the individuals an adequate mobile Internet QoE.
\end{abstract}

Index Terms : mobile, network, datasets, prediction

\section{INTRODUCTION}

Web use on mobile devices makes it possible for the mobile world wide web clients (i.e., consumers) to associate with friends and family via vocal or online video calls, web browsing, e-mail, sharing of photos as well as other info through the accessible mobile functions mounted on individuals " mobile phones. Considering that, mobile world wide web users have actually ended up being the main concentration of service style for the mobile network operators, quality of experience is right now a brand-new phrase coined to be used due to the MNOs to quantify, manage, as well as strengthen the understanding of the mobile network users. The vast use of web located services has been outlined up to the development of a fast mobile network situated on Universal Mobile Telecommunication Solutions, Lasting Advancement, and also various other telecommunications (Telecoms) criteria. Similarly, the supply of more significant information transmission velocity (throughput) allows mobile internet consumers to transcend web searching through permitting solutions, including report transfer, report download, online video streaming, and also a voice-over web protocol.

Revised Manuscript Received on December 30, 2019.

* Correspondence Author

Damera Priyanka, Faculty, Department of IT, Kakatiya University College of Engineering and Technology, Warangal, India.

Mamidala Soujanya, Faculty, Department of IT, Kakatiya University College of Engineering and Technology, Warangal, India.

India

Syed Abdul Moeed, Assistant Professor, Department of CSE, Kakatiya Institute of Technology and Science, Warangal, India.

(C) The Authors. Published by Blue Eyes Intelligence Engineering and Sciences Publication (BEIESP). This is an open access article under the CC BY-NC-ND license (http://creativecommons.org/licenses/by-nc-nd/4.0/)

Nevertheless, the mobile network operators have a tendency to restrict the existing data-rate manageable due to the consumers as a result of the high price associated with obtaining range [1] Simultaneously, the rise in the number of users have actually enriched competitive advantage and provision of inexpensive solutions, thereby imposing extra problem on the MNOs in providing a sufficient degree of service functionality to the mobile internet users. Specifically, mobile networks are susceptible to direct supply (including lessened channel schedule) that dynamically transforms overtime because of the nearby congestion, which commonly lead in to endangering the consumer session [4] The said instances, increase in restricted data fee as well as regional blockage can truly have impact on the mobile web individual experience.

It is every bit as important to bear in mind that the desire of the mobile internet consumers is based on discovered experiences from the network performance, which are customarily expected to be secure and less congested. To allow a satisfactory level of consumer experience, the MNOs are actually needed to be properly updated regarding the visitor traffic characteristics triggered by the geographical as well as dynamic attributes of the network traffic [1] Comprehending the network traffic characteristics will allow the MNOs to plan as well as maximized the NP to recognize the topographical as well as temporal service-related Quality of Experience from both the individuals' as well as network perspectives.

However, QoS is not especially related to the user's understanding [2], as such QoS alone wants to determine the mobile world wide web users " viewpoint [3] QoE is actually a multidimensional concept that includes both the individual and unbiased aspects of the world wide web services offered by the MNOs [3] On one palm, the very subjective aspect of web solutions provided due to the MNOs targeted at subscribers " knowledge, expectation, personal as well as social background [3] While, the unbiased aspect of the net services is actually the network performance frequently embodied in the form of the QoS specifications (e.g., throughput, data transfer, loss, problem and also jitter). QoE has actually come to be a vital variable for the majority of mobile web customers when opting for aMNO. For that reason, the MNOs are actually faced with the obstacles of surveillance and gauging QoE, considering that the efficiency of individual MNO can vary between site, time of the day, as well as may certainly not always comply with the mobile internet customers desires. Sizable volume of literature has actually proposed and also evaluated internet QoE by means of individual and also unbiased methods in each fixed-server made use of on home computer and smartphones [4] 
The subjective procedure of gauging QoE is a type of poll generally performed in a laboratory experiments using mean opinion credit rating that stands for the individual QoE of the internet clients for a particular network company. Several research studies have used MOS to measure the QoE of different worldwide web services provided due to the MNOs, the likes of online video streaming [5], Voice over Net Process, Skype voice telephone calls as well as web-browsing. Come from these research studies displayed that the proper technique is an important dimension considering that it comprised the deliberate as well as unconscious aspects of the subscriber's quality examination method that may undoubtedly not otherwise be caught. Regardless of the dependability benefit of a very subjective dimension, previous studies have mentioned that the very personal size is pricey, taxing, not reproducible on demand as well as might certainly not be adequate for in-service quality tracking. The setbacks linked with the very subjective measurement create the objective size, which may gauge as well as predict the web QoE in genuine or close to real-time. [7] To facilitate the QoE reasoning coming from encrypted video sessions, our experts build an experimental framework with automatic streaming and also a compilation of network signs and also ground reality of video treatments, as well as QoE statistics estimate. Our company uses this structure to do a considerable assessment of eMIMIC with a pair of prominent commercial online video streaming solutions. On top of that, our experts imitate a recently made a proposal device learning-based QoE estimate approach, hereon referred to as ML16, by totally implementing and also using it to the same pair of video clip companies. This assists in knowing the distinctions in functionality and also precision in between both QoE estimation methods, to ensure they may be further developed and also boosted.

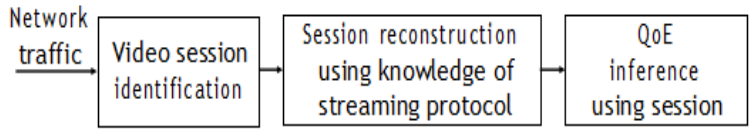

(a) Overview of SM-based approach

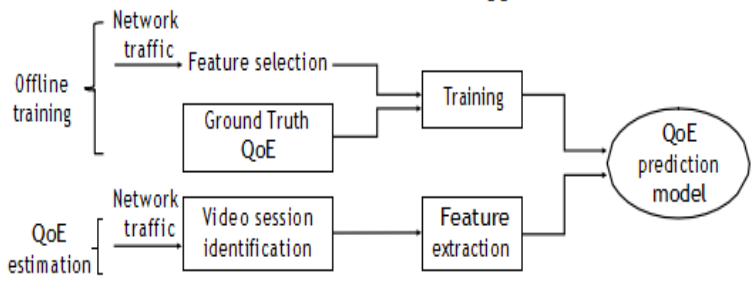

(b) Overview of ML-based app roach

Figure 1 : Inference approaches of QoE

\section{QoE MOBILE INTERNET}

In wireless communication area, mobile world wide web is among the most fast-growing area due to its influence in folks " every day life and enormous profit produced due to the MNOs. The development was actually connected with the huge advancement of different web applications utilized on smart phones. QoE is a crucial period of mobile web company provisioning because of the spontaneous development of clients who access the internet through their cell phones. According to Qualinet white paper in [7], QoE is referred to as the "degree of joy or even annoyance of the consumer of an app or service. It results from the fulfilment of his or her desires with respect to the electrical and also/ or even fulfillment of the application or even solution in the lighting of the consumer " $\mathrm{s}$ character and also present condition." This definition signifies QoE incorporates the user's belief, assumptions, network performance and experience of the service apps. Mobile net companies offered by the MNO is actually determined through various QoE factors that is system (e.g., problem, throughput, jitter, loss and also security), individual (gender, age, history and also learning), and also situation (area, time of the time, prices and also registration kind. Evaluated QoE influence aspects promotes the analysis of users identified QoE. Device QoE impact think about the type of QoS specifications (throughput, reduction, data transfer, hold-up, and also jitter) is generally reviewed in the literature. One of the QoS specifications, problem, jitter and also latency is the best analyzed in previous researches, given that previous researches felt quality degration influences the customers " experience. While many research studies in the business of QoE has assessed throughput size for both cordless web website traffic and mobile web applications, handful of research studies have utilized aggregated throughput measurements experienced due to the consumer "s mobile network atmosphere. This signifies that, previous research studies taken a look at QoEdegration with the vital network information and also performance dataset compiled in laboratory experiments by means of the desktop.

\section{RESULT : PROPOSED MODELLING MOBILE NETWORK PERCEIVED QoE FRAMEWORK}

Modelling of viewed QoE is actually worried about the process of forecasting the identified QoE of consumers via a theoretical symbol of information and also its connection coming from the details collected coming from the customers, network, or even each customers' networks, taking into consideration the drawbacks of context as well as content of the company noted in the unbiased measurement of identified QoE, restricted use of huge and diverse dataset produced in mobile networks specified in previous parts. Furthermore, based upon the obstacles encountered by the MNOs in studying the huge volume of data made up in the mobile network, this section proposed a framework that permits modelling of viewed QoE in the mobile network via the significant data analytics approach, considering that the MNOs might use significant records analytics to possess a clear and current understanding of the individuals experience to permit all of them measure and also model recognized QoE of the mobile World wide web consumers. The recommended platform explains the method of acquiring information from the mobile network web traffic and also the 3 procedures of major records analytics in the real time dimension platform as depicted in Figure 2. 


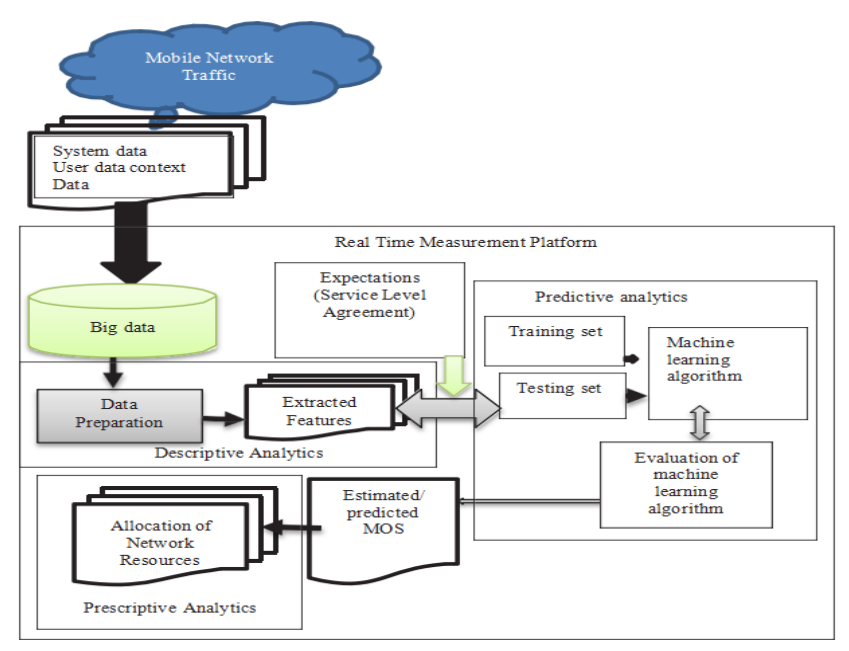

Figure 2 : Framework for modelling perceived QoE

\begin{tabular}{|c|c|c|}
\hline Category & $\mathrm{KQl}$ & KPI \\
\hline Service availability & \% of service downtime & Session setup success ratio \\
\hline \multirow{3}{*}{ Service responsiveness } & Session start delay & Setup delay \\
\hline & Pause delay & Control signal delay \\
\hline & Fasstforward rewind delay & Control signal delay \\
\hline \multirow{3}{*}{ Service quality } & Video MOS score (MOSV) & Blockiness, jerkiness, buriness \\
\hline & Audio MOS score (MOSa) & Delay, jitter, packet loss \\
\hline & $\begin{array}{l}\text { Combined AN quality (MOSav) audiotvideo } \\
\text { synchronization (Iip synch) }\end{array}$ & Delay, iltter, packet loss \\
\hline $\begin{array}{l}\text { AN-Auviolisisul } \\
\text { XPl-Key performance indiciator }\end{array}$ & $\begin{array}{l}\text { KQ1-Key yudityinidict: } \\
\text { MOS-Mean oponion so }\end{array}$ & \\
\hline
\end{tabular}

Table 1 : KPI/KQI

The KPI and KQI are typically removed through the pre-processing of the raw dataset compiled coming from different network components and probes. KPI and also KQI are removed coming from the pre-processing method because the dataset contained in mobile network visitor traffic is actually assumed to become inconsistent as well as filthy as a result of the large attribute of the dataset. Additionally, huge data making up the KPI and also KQI are actually usually offered in an unstructured kind that might not agree with for the modelling of perceived QoE. The records pre-processing period of the major data analytics will ensure dependability, efficiency, randomness, as well as consistency of the dataset to make it appropriate for the perceived QoE modelling period. In many cases, dependability of the dataset will definitely make sure the represented dataset is actually accurate good enough to suit the identified QoE modelling stage. The randomness of the datasets illustrates the statistical qualities of the total datasets, which is extremely essential for prolegomenous information evaluation as well as visual images of the dataset. After that the consistency of the data will definitely make certain the dataset create the same end result within a reasonable inaccuracy scope when a various random sample evaluation is performed. Within this case, use of exploratory data evaluation and also conventional data pre-processing strategies such as records cleaning, records integration, information decline and information transformation are actually frequently used in the data-mining technique; function selection and also removal will properly support the big analytics methodology to assist the method of modelling recognized QoE of the mobile Internet individuals. Consequently, the recommended platform embraces making use of huge information obtained coming from the mobile network traffic including several KPI as well as KQI, which work with the regarded QoE impact elements as the primary foundation for modelling viewed QoE of mobile Web service-related treatments.

It deserves pointing out that using requirement such as service degree contract is actually a crucial parameter for modelling identified QoE, but the use of SLA is still limited in the literature. The typical procedure for making use of expectation in modelling viewed QoE is by talking to the customers what is actually expected from the MNOs through the process of a poll. Yet considering the moment consuming as well as expensive nature of the subjective strategy used in gathering personal consumer assumptions, individual measurement may certainly not appropriate in large-scale settings. Additionally, the subjective procedure does not have repeatability and is actually not effective in real-time cases. Nevertheless, when it comes to the goal method where the users' experience would be captured as well as analyzed in real-time without direct feedback from the individuals' it is critical to make use of SLA in addition to various other QoE influence variables to approximate the customers regarded QoE. SHANTY TOWN is the contract between the customer and also the MNOs on service characteristics, like company amount purposes, company surveillance components and also monetary remuneration parts. The telecoms regulators commonly use RUN-DOWN NEIGHBORHOOD to determine the whether the services delivered by the MNOs observe the requirements stated in the contract. Consequently, SKID ROW is actually integrated in the recommended structure as proposed in the current studies. Hiring SLA in the suggested platform for creating perceived QoE would help the MNOs to figure out when several variables perform not fulfill the expected level mentioned in the SLA and just how exactly the variables included influence individual experience. Overall, making use of SLA as individual desire in modelling regarded QoE would assist the procedure of figuring out the anticipated MOS, based on the optimum as well as minimum required values mentioned in the SLA. The prescribed analytics capitalizes on the end results gotten from both the definitive and predictive analytics to choose the greatest selection or action that may be needed to strengthen the NP of the mobile network. As suggested in Figure 2, this article recommended the forecasted MONTH may be used for suitable appropriation network resources in sites where the MOS is actually below assumptions. Conclusively, the use of significant records analytics to take care of the perceived QoE can easily make it possible for the MNOs to deliver proactive measures prior to the consumers will identify any network distortion while utilizing the Net solutions provided by the MNOs.

Published By:

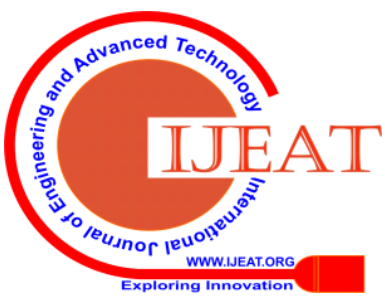


In addition, it may aid the MNOs to take optimal choices for efficient management of their NP to make it possible for a better arrangement of the Internet services.

An abstract depiction as well as partnership of dataset gathered coming from the consumers, network, or each and analysed with analytical as well as algorithms software program for automated prophecy to receive a greater precision is the method of modelling seen QoE. Based on the setbacks of minimal use mobile network diverse dataset including the context as well as material of the web solutions highlighted in the previous research studies. The enhanced regarded QoE platform aimed at anticipating the viewed QoE by means of the identified QoE impact elements, perceived QoE dimensions and also estimates along with the objective of eliminating limited use of mobile net usage data including throughput metrics, correlating context (place and also time of the day), web content of the services, requirement from both the consumer as well as the $\mathrm{MNO}^{\text {"e }} \mathrm{s}$ point of view are actually still limited in the literary works.

Numerous researches have indicated that contextual experiences a straight influence identified Consequently, evaluation of recognized QoE was actually based on all the 3 dimensions of the identified QoE effect variables, which permits an enough evaluation of the regarded QoE in connection with mobility (like opportunity as well as location). The viewed QoE platform was designed to get over the downsides related to the subjective size as well as generate the objective measurement, which can gauge and anticipate the world wide web QoE in true or even close to real-time without human intervention and also enhance in-service quality monitoring. Additionally, the platform permits the prediction of regarded QoEthrough the use of mobile web use records containing throughput metrics, correlating situation (place and opportunity of the time), material of the companies, expectation coming from both the user as well as the MNO " $\mathrm{s}$ viewpoint that is limitedly examined in the literature. The viewed QoE modelling framework is actually portrayed in Figure 3.

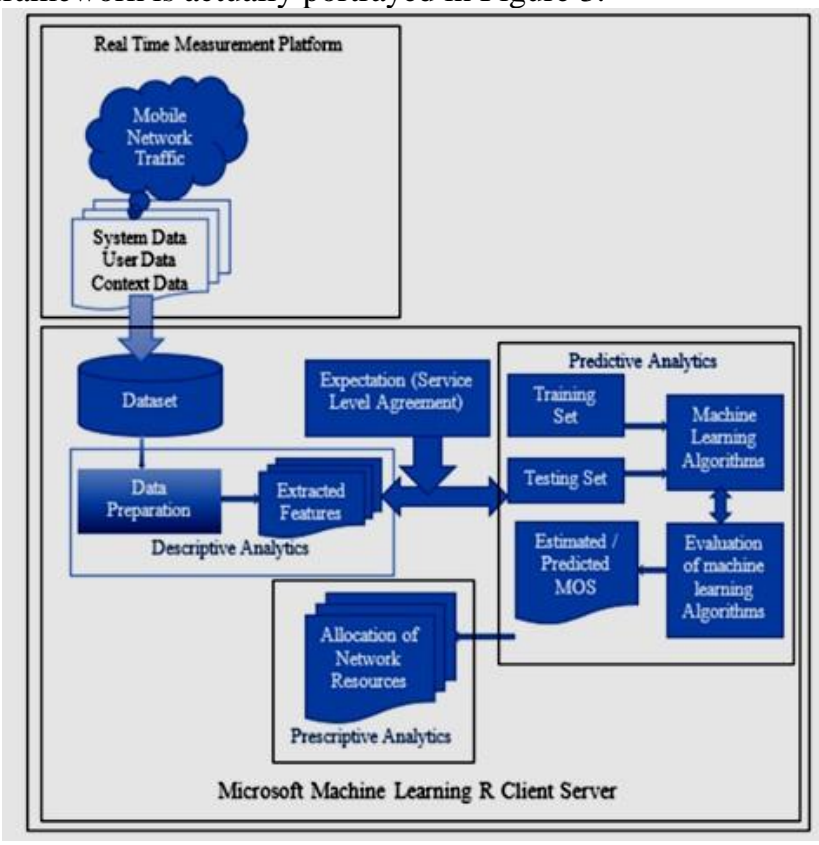

Figure 3 : QoE Prediction

\section{CONCLUSION}

To assist in the comprehensive examination, our experts establish a speculative structure that permits digital streaming as well as an assortment of network signs and ground reality QoE metrics of two prominent video clip service providers. This study provided a summary of mobile web QoE, boosted the identified QoE modeling structure proposed in a previous research study. Today structure highlighted the relevance of making use of datasets compiled from the mobile network website traffic, as the datasets assisted multiple situations and service-related metrics for accurate modeling of the mobile Web recognized QoE.

\section{REFERENCES}

1. B. A. A. Nunes, M. Mendonca, X.-N. Nguyen, K. Obraczka, and also T. Turletti, "A questionnaire of software-defined media: Past, existing, and also future of programmable networks," IEEE Communications Studies \& Tutorials, vol. 16, no. 3, pp. 1617-- 1634, 2014

2. G. Dimopoulos, I. Leontiadis, P. Barlet-Ros, as well as K Papagiannaki, "Assessing Online Video QoE from Encrypted Traffic," in Proc. ACM IMC, 2016.

3. H. Nam, K.-H. Kim, as well as H. Schulzrinne, "Qoe matters greater than qos: Why folks cease viewing pet cat video recordings," in Proc. IEEE INFOCOM, 2016

4. T. Stockhammer, "Dynamic Adaptive Streaming over HTTP Standards as well as Style Guidelines," in Proc. ACM MMSys, 2011.

5. J. J. Ramos-Mun oz, J. Prados-Garzon, P. Ameigeiras, J. Navarro-Ortiz, as well as J. M. Lo' pez-Soler, "Qualities of Mobile YouTube Traffic," IEEE Wireless Communications, vol. 21, no. 1, pp. 18-- 25, 2014.

6. A. Mansy, M. Ammar, J. Chandrashekar, as well as A. Sheth, "Characterizing Customer Habits of Industrial Mobile Video Clip Streaming Companies," in Proceedings of Sessions on Mobile Online Video Distribution. ACM, 2014, p. 8.

7. C. Sieber, A. Blenk, M. Hinteregger, and W. Kellerer, "The Price of Aggressive HTTP Adaptive Streaming: Measuring YouTube's Redundant Web traffic," in 2015 IFIP/IEEE International Seminar on Integrated System Management (IM). IEEE, 2015, pp. 1261-- 1267.

8. Shoban Babu Sriramoju, "An Application for Annotating Web Search Results" in "International Journal of Innovative Research in Computer and Communication Engineering" Vol 2,Issue 3,March 2014 [ ISSN(online) : 2320-9801, ISSN(print) : 2320-9798 ]

9. Ajay Babu Sriramoju, Dr. S. Shoban Babu, "Analysis on Image Compression Using Bit-Plane Separation Method" in "International Journal of Information Technology and Management", Vol VII, Issue X, November 2014 [ ISSN : 2249-4510 ]

10. Anusha Medavaka, Dr.P.Niranjan, P. Shireesha, "USER SPECIFIC SEARCH HISTORIES AND ORGANIZING PROBLEMS" in "International Journal of Advanced Computer Technology (IJACT)", Vol. 3, Issue No. 6 [ISSN : 2319-7900]

11. Monelli Ayyavaraiah, Shoban Babu Sriramoju, "A Survey on the Approaches in Targeting Frequent Sub Graphs Mining" in "Indian Journal of Computer Science and Engineering (IJCSE)", Volume 9 Issue 2, Apr-May 2018 [e-ISSN : 0976-5166 p-ISSN : 2231-3850], DOI : $10.21817 /$ indjcse/2018/v9i2/180902024

12. Anusha Medavaka, P. Shireesha, "A Survey on TraffiCop Android Application" in "Journal of Advances in Science and Technology", Vol. 14, Issue No. 2, September-2017 [ISSN : 2230-9659]

13. Anusha Medavaka,"Monitoring and Controlling Local AreaNetwork Using Android APP" in "International Journal of Research", Vol. 7, Issue No. IV, April-2018 [ISSN : 2236-6124]

14. Mounica Doosetty, Keerthi Kodakandla, Ashok R, Shoban Babu Sriramoju, "Extensive Secure Cloud Storage System Supporting Privacy-Preserving Public Auditing" in "International Journal of Information Technology and Management", Volume VI, Issue I, Feb 2012 [ ISSN : 2249-4510 ]

15. Anusha Medavaka, "Algorithm Feasibility on IoT Devices with Memory and Computational Power Constraints", International Journal of Science and Research (IJSR), Volume 8, Issue 5, May 2019[ISSN : 2319-7064] 


\section{AUTHORS PROFILE}

Damera Priyanka, working as a faculty in department of IT at Kakatiya University College of Engineering and Technology. My research interests are mobile networks and IOT.

Mamidala Soujanya, working as a faculty in department of IT at Kakatiya University College of Engineering and Technology. My research interests are mobile networks, IOT and Data Mining.

Syed Abdul Moeed, working as a faculty in department of IT at Kakatiya University College of Engineering and Technology. My research interests are mobile networks, IOT and Network Security. 